\title{
Catalytic Enantioselective Sulfinyl Transfer Using Cinchona Alkaloid Catalysts
}

\author{
Hillary M. Peltier, Jared W. Evans, Jonathan A. Ellman* \\ Center for New Directions in Organic Synthesis, Department of Chemistry, University of California, Berkeley, \\ California 94720
}

\section{Supporting Information}

Reagents and General Methods. Unless otherwise noted, all starting materials were obtained from commercial suppliers and used without further purification. $1,8-$ Bis(dimethylamino)naphthalene was recrystallized from absolute EtOH. tert-Butylsulfinyl chloride was prepared using the previously published method. ${ }^{1}$ Tetrahydrofuran (THF) was distilled from sodium/benzophenone ketyl immediately prior to use. $\mathrm{CH}_{2} \mathrm{Cl}_{2}$, acetonitrile $\left(\mathrm{CH}_{3} \mathrm{CN}\right)$, and triethylamine $\left(\mathrm{Et}_{3} \mathrm{~N}\right)$ were distilled from $\mathrm{CaH}_{2}$ immediately prior to use. Toluene was distilled from $\mathrm{Na}$. The concentration of commercially available solutions of phenyllithium in cyclohexane-ether was determined by titration with 2,6-di-tert-butyl-4-methylphenol and 1,10-phenanthroline. Reactions were carried out in flame- or oven- dried glassware under a $\mathrm{N}_{2}$ atmosphere. Extracts were dried over $\mathrm{Na}_{2} \mathrm{SO}_{4}$ and concentrated using a Buchi rotary evaporator under reduced pressure. Chromatography was carried out with Merck $60 \AA$ 230-400 mesh silica gel. IR spectra were recorded on a Nicolet Avatar 360 FTIR spectrometer equipped with an attenuated total reflectance accessory and only partial data are listed. ${ }^{1} \mathrm{H}$ and ${ }^{13} \mathrm{C}$ NMR spectra were obtained in $\mathrm{CDCl}_{3}$ at room temperature. Chemical shifts in NMR spectra are expressed in ppm relative to $\mathrm{CDCl}_{3}$. Coupling constants are reported in $\mathrm{Hz}$. Enantiopurities were determined by HPLC using Diacel CHIRALCEL OD, CHIRALCEL AD, and CHIRALCEL AS (4.6 x 250 $\mathrm{mm}$ ) columns with UV detection at 210 and $254 \mathrm{~nm}$. Elemental analyses were performed by the University of California at Berkeley Micro-Mass Facility. Spectroscopic data of sulfinate esters

19a and 19c is consistent with previously published data. ${ }^{1}$ 


\section{General Procedure A: Enantioselective formation of sulfinate esters (2, 19a-g). A}

solution of proton sponge ( $54.0 \mathrm{mg}, 0.250 \mathrm{mmol}, 2.50$ equiv), alcohol ( $0.500 \mathrm{mmol}, 5.00$ equiv), catalyst ( $0.0100 \mathrm{mmol}, 0.100$ equiv), and internal standard, 2,6-dimethoxytoluene (15.2 mg, $0.100 \mathrm{mmol}, 1.00$ equiv) in the indicated solvent $(0.500 \mathrm{~mL}, 0.200 \mathrm{M})$ was cooled to the indicated temperature and sulfinyl chloride $1(50.0 \mu \mathrm{L}, 0.100 \mathrm{mmol}, 2.00 \mathrm{M})$ was added as a

solution in toluene over a period of $5 \mathrm{~min}$. At the indicated time, $2.00 \mathrm{~mL}$ of $\mathrm{NEt}_{3} / \mathrm{CD}_{3} \mathrm{OD}(0.20$ $\mathrm{mL}$ of $\mathrm{NEt}_{3} / 10 \mathrm{~g} \mathrm{CD}_{3} \mathrm{OD}$ ) was added to quench any remaining 1 and $0.300 \mathrm{~mL}$ of the mixture was transferred to an NMR tube containing $1.00 \mathrm{~mL}$ of $\mathrm{CDCl}_{3}$. After yield determination by NMR integration relative to the internal standard, the solvents were removed in vacuo and the residue was diluted with hexanes (ca. $3.00 \mathrm{~mL}$ ) and filtered for HPLC analysis. (For yields and ee, see Tables 1-4. See experimental procedures for the preparation, characterization, and chiral HPLC conditions for the racemic sulfinate esters listed below.)

\section{General Procedure B: Formation of racemic sulfinate esters $(2,19 b, 19 d, 19 e, 19 f$,} 19g). A solution of the alcohol ( $4.45 \mathrm{mmol}, 5.00$ equiv) and $\mathrm{NEt}_{3}(0.186 \mathrm{~mL}, 1.33 \mathrm{mmol}, 1.50$ equiv) in THF ( $4.45 \mathrm{~mL}, 0.200 \mathrm{M})$ was cooled to $-78{ }^{\circ} \mathrm{C}$. Sulfinyl chloride $1(0.404 \mathrm{~mL}, 0.889$ $\mathrm{mmol}, 2.20 \mathrm{M}, 1.00$ equiv) was added as a solution in toluene, and after $2 \mathrm{~h}$ the reaction vessel was removed from the cold bath. Once the reaction mixture warmed to room temperature, it was stirred for $1 \mathrm{~h}$, diluted with EtOAc $(50 \mathrm{~mL})$, and washed with $1 \mathrm{~N} \mathrm{HCl}(50 \mathrm{~mL}), \mathrm{NaHCO}_{3}(50$ $\mathrm{mL})$, brine $(50 \mathrm{~mL})$, and then dried and filtered. The EtOAc was removed in vacuo, and the residue was purified via silica gel chromatography to afford pure sulfinate ester.

2-Methylpropane sulfinic acid benzyl ester (2). Reaction of $0.461 \mathrm{~mL}(4.45 \mathrm{mmol})$ of benzyl alcohol according to General Procedure B yielded pure $2(0.162 \mathrm{~g}, 86 \%)$ as a colorless oil after chromatography (10\% EtOAc/hexanes) with spectral data corresponding to the literature 
report. ${ }^{1}$ HPLC (Diacel Chiralpak OD column, 96:04 hexanes/IPA; $\left.1.0 \mathrm{~mL} / \mathrm{min}\right) \mathrm{t}_{1}=7.6 \mathrm{~min}, \mathrm{t}_{2}=$ $8.7 \mathrm{~min} .{ }^{1} \mathrm{H}$ NMR (400 MHz) $\delta 1.20(\mathrm{~s}, 9 \mathrm{H}), 4.99(\mathrm{~d}, 1 \mathrm{H}, J=11.8 \mathrm{~Hz}), 5.13(\mathrm{~d}, 1 \mathrm{H}, J=11.8$ $\mathrm{Hz}), 7.31(\mathrm{~m}, 5 \mathrm{H})$.

2-Methylpropane-2-sulfinic acid 4-chlorobenzyl ester (19b). Reaction of $0.635 \mathrm{~g}$ (4.45 mmol) of 4-chlorobenzyl alcohol according to General Procedure B yielded pure 19b $(0.217 \mathrm{~g}, 99 \%)$ as a colorless oil after chromatography (25\% EtOAc/hexanes). HPLC (Diacel Chiralpak OD column, 96:04 hexanes/IPA; $1.0 \mathrm{~mL} / \mathrm{min}$ ) $\mathrm{t}_{1}=7.9 \mathrm{~min}, \mathrm{t}_{2}=9.9 \mathrm{~min}$. IR 3053, 2959, 1599, 1493, 1393, $1127 \mathrm{~cm}^{-1} .{ }^{1} \mathrm{H}$ NMR (400 MHz) $\delta 1.20(\mathrm{~s}, 9 \mathrm{H}), 4.95(\mathrm{~d}, 1 \mathrm{H}, J=12.0$ $\mathrm{Hz}), 5.09(\mathrm{~d}, 1 \mathrm{H}, J=12.0 \mathrm{~Hz}), 7.33(\mathrm{~m}, 4 \mathrm{H}) .{ }^{13} \mathrm{C} \mathrm{NMR}(100 \mathrm{MHz}) \delta 21.4,57.4,69.8,128.5$, 129.3, 134.1, 134.4. Anal. Calcd for $\mathrm{C}_{11} \mathrm{H}_{15} \mathrm{ClO}_{2} \mathrm{~S}$ : C, 53.54; H, 6.13. Found: C, 53.94; H, 6.27. 2-Methylpropane-2-sulfinic acid naphthalen-1-ylmethyl ester (19d). Reaction of $0.703 \mathrm{~g}$ (4.45 mmol) of 1-naphthylmethanol according to General Procedure B yielded pure 19d $(0.200 \mathrm{~g}, 86 \%)$ as a colorless oil after chromatography (2:1 hexanes/EtOAc). HPLC (Diacel Chiralpak OD column, 96:04 hexanes/IPA; $1.0 \mathrm{~mL} / \mathrm{min}) \mathrm{t}_{1}=11.6 \mathrm{~min}, \mathrm{t}_{2}=13.7 \mathrm{~min} . \quad$ IR 3053, 2963, 2869, 1600, 1510, 1477, 1362, $1119 \mathrm{~cm}^{-1}$. ${ }^{1} \mathrm{H}$ NMR (500 MHz) $\delta 1.14(\mathrm{~s}, 9 \mathrm{H}), 5.36(\mathrm{~d}$, $1 \mathrm{H}, J=11.8), 5.62(\mathrm{~d}, 1 \mathrm{H}, J=11.8), 7.43-7.46(\mathrm{~m}, 1 \mathrm{H}), 7.50-7.58(\mathrm{~m}, 3 \mathrm{H}), 7.85-7.88(\mathrm{~m}, 2 \mathrm{H})$, $8.08(\mathrm{~d}, 1 \mathrm{H}, J=8.5 \mathrm{~Hz}) .{ }^{13} \mathrm{C} \mathrm{NMR}(125 \mathrm{MHz}) \delta 21.6,57.5,69.1,123.8,125.2,126.0,126.6$, 127.7, 128.6, 129.7, 131.5, 131.6, 133.7. Anal. Calcd for $\mathrm{C}_{15} \mathrm{H}_{18} \mathrm{O}_{2} \mathrm{~S}: \mathrm{C}, 68.67 ; \mathrm{H}, 6.92$. Found: C, 68.93; H, 7.14 .

2-Methylpropane-2-sulfinic acid 2,6-dichlorobenzyl ester (19e). Reaction of $0.787 \mathrm{~g}$ (4.45 mmol) of 2,6-dichlorobenzyl alcohol according to General Procedure B yielded pure 19e $(0.232 \mathrm{~g}, 93 \%)$ as a white crystalline solid after chromatography (25\% EtOAc/ hexanes). HPLC (Diacel Chiralpak AD column, 96:04 hexanes/IPA 1.0mL/min) $\mathrm{t}_{1}=7.6 \mathrm{~min}, \mathrm{t}_{2}=8.1 \mathrm{~min}$. IR 
$3027,2959,1591,1363,1125 \mathrm{~cm}^{-1} .{ }^{1} \mathrm{H}$ NMR $(500 \mathrm{MHz}) \delta 1.16(\mathrm{~s}, 9 \mathrm{H}), 5.32(\mathrm{~d}, 1 \mathrm{H}, J=11.3$

$\mathrm{Hz}), 5.37(\mathrm{~d}, 1 \mathrm{H}, J=11.3 \mathrm{~Hz}), 7.25-7.37(\mathrm{~m}, 3 \mathrm{H}) .{ }^{13} \mathrm{C}$ NMR $(100 \mathrm{MHz}) \delta 21.4,57.6,65.3$,

128.4, 130.7, 131.7, 136.8. Anal. Calcd for $\mathrm{C}_{11} \mathrm{H}_{14} \mathrm{Cl}_{2} \mathrm{O}_{2} \mathrm{~S}: \mathrm{C}, 46.98 ; \mathrm{H}, 5.02$. Found: $\mathrm{C}, 47.08$;

H, 5.20.

2-Methylpropane-2-sulfinic acid 2,6-methylbenzyl ester (19f). Reaction of $0.606 \mathrm{~g}$ (4.45 mmol) of 2,6-dimethylbenzyl alcohol ${ }^{2}$ according to General Procedure B yielded pure 19f $(0.210 \mathrm{~g}, 98 \%)$ as a colorless oil after chromatography (3:1 hexanes/EtOAc). HPLC (Diacel Chiralpak OD column, 99:01 hexanes/IPA; $1.0 \mathrm{~mL} / \mathrm{min}) \mathrm{t}_{1}=9.3 \mathrm{~min}, \mathrm{t}_{2}=10.8 \mathrm{~min}$. IR 3075, 2958, 1562, 1438, $1123 \mathrm{~cm}^{-1} .{ }^{1} \mathrm{H}$ NMR (400 MHz) $\delta 1.15(\mathrm{~s}, 9 \mathrm{H}), 2.42(\mathrm{~s}, 6 \mathrm{H}), 5.08(\mathrm{~d}, 1 \mathrm{H}, J=$ $11.1 \mathrm{~Hz}), 5.17(\mathrm{~d}, 1 \mathrm{H}, J=11.1 \mathrm{~Hz}), 7.06(\mathrm{~d}, 2 \mathrm{H}, J=7.5 \mathrm{~Hz}), 7.17(\mathrm{t}, 1 \mathrm{H}, J=7.6 \mathrm{~Hz}) .{ }^{13} \mathrm{C}$ NMR $(100 \mathrm{MHz}) \delta 19.6,21.5,57.3,65.1,128.2,128.9,132.2,138.3$. Anal. Calcd for $\mathrm{C}_{13} \mathrm{H}_{20} \mathrm{O}_{2} \mathrm{~S}: \mathrm{C}$, 64.96; H, 8.39. Found: C, 64.99; H, 8.67.

2-Methylpropane-2-sulfinic acid 2,4,6-trichlorobenzyl ester (19g). Reaction of 0.940 $\mathrm{g}$ (4.45 mmol) of 2,4,6-trichlorobenzyl alcohol according to General Procedure B yielded $\mathbf{1 9 g}$ $(0.224 \mathrm{~g}, 80 \%)$ as a white crystalline solid after chromatography (3:1 hexanes/EtOAc). HPLC (Diacel Chiralpak AD column, 96:04 hexanes/IPA; $1.0 \mathrm{~mL} / \mathrm{min}$ ) $\mathrm{t}_{1}=6.5 \mathrm{~min}, \mathrm{t}_{2}=7.0 \mathrm{~min}$. IR 3064, 2959, 1582, 1550, 1459, 1380, $1131 \mathrm{~cm}^{-1} .{ }^{1} \mathrm{H}$ NMR (500 MHz) $\delta 1.15(\mathrm{~s}, 9 \mathrm{H}), 5.25(\mathrm{~d}$, $1 \mathrm{H}, J=11.4 \mathrm{~Hz}), 5.30(\mathrm{~d}, 1 \mathrm{H}, J=11.4 \mathrm{~Hz}), 7.36(\mathrm{~s}, 2 \mathrm{H}) .{ }^{13} \mathrm{C}$ NMR $(125 \mathrm{MHz}) \delta 21.5,57.8$, 64.7, 128.5, 130.6, 135.8, 137.4. Anal. Calcd for $\mathrm{C}_{11} \mathrm{H}_{13} \mathrm{Cl}_{3} \mathrm{O}_{2} \mathrm{~S}$ : C, 41.86; H, 4.15. Found: $\mathrm{C}$, 42.23; H, 4.27.

TBDPS-S-(-)-1-Methyl-2-pyrrolidinemethanol (5). To a solution of (S)-(-)-1methyl-2-pyrrolidinemethanol $(0.0673 \mathrm{~mL}, 0.566 \mathrm{mmol})$ and imidazole $(73.2 \mathrm{mg}, 1.07 \mathrm{mmol})$ in $\operatorname{DMF}(0.210 \mathrm{~mL}, 2.70 \mathrm{M})$ at $0{ }^{\circ} \mathrm{C}$ was added tert-butylchlorodiphenyl silane $(0.177 \mathrm{~mL}, 0.679$ 
mmol). The ice bath was removed after $15 \mathrm{~min}$ and the mixture was stirred at $\mathrm{rt}$ for $20 \mathrm{~h}$. The reaction mixture was diluted with $\mathrm{EtO}_{2}(10 \mathrm{~mL})$ and washed with $\mathrm{H}_{2} \mathrm{O}(3 \times 10 \mathrm{~mL})$ and brine $(1$ x $10 \mathrm{~mL})$. The organic layer was dried, filtered, and concentrated. Column chromatography (2:1 hexanes/EtOAc) afforded $5(69.4 \mathrm{mg}, 35 \%)$ as a colorless oil. IR 3071, 2930, 2854, $2771 \mathrm{~cm}^{-1}$. ${ }^{1} \mathrm{H}$ NMR $(500 \mathrm{MHz}) \delta 1.07$ (s, 9H), 1.53-1.59 (m, 1H), 1.67-1.78 (m, 2H), 1.89-1.96 (m, 1H), 2.20-2.24 (m, 1H), 2.37-2.42 (m, 1H), $2.39(\mathrm{~s}, 3 \mathrm{H}), 3.01-3.05(\mathrm{~m}, 1 \mathrm{H}), 3.53(\mathrm{dd}, 1 \mathrm{H}, J=10.1$, 6.3), $3.75(\mathrm{dd}, 1 \mathrm{H}, J=10.1,5.7), 7.38-7.44(\mathrm{~m}, 6 \mathrm{H}), 7.68-7.70(\mathrm{~m}, 4 \mathrm{H}) .{ }^{13} \mathrm{C} \mathrm{NMR}(125 \mathrm{MHz}) \delta$ $19.2,22.7,26.8,28.7,41.9,57.9,66.9,67.8,127.6,129.5,133.7,133.8,135.58,135.6 . \mathrm{MS}$ (FAB): $m / z 354\left(\mathrm{MH}^{+}\right)$. HRMS (FAB) Calcd for $\left(\mathrm{MH}^{+}\right)$: 354.2253. Found 354.2248.

\section{Large scale procedure for the enantioselective formation of sulfinate $19 \mathrm{~g}$ with $\mathbf{1 0}$}

mol\% of quinidine. A solution of proton sponge (846 mg, $3.95 \mathrm{mmol}, 2.50$ equiv), 2,4,6trichlorobenzyl alcohol (1.67 g, $7.90 \mathrm{mmol}, 5.00$ equiv), and quinidine (51.3 mg, $0.158 \mathrm{mmol}$, 0.100 equiv) in THF $(7.90 \mathrm{~mL}, 0.200 \mathrm{M})$ was cooled to $-40{ }^{\circ} \mathrm{C}$ (internal temperature) and sulfinyl chloride $\mathbf{1}(0.790 \mathrm{~mL}, 1.58 \mathrm{mmol}, 2.00 \mathrm{M})$ was added as a solution in toluene over a period of $40 \mathrm{~min}$. After $20 \mathrm{~h}, 10 \mathrm{~mL}$ of $\mathrm{MeOH}$ and $0.2 \mathrm{~mL}$ of $\mathrm{Et}_{3} \mathrm{~N}$ were added and the reaction solution was stirred for $1 \mathrm{~h}$ at $-40{ }^{\circ} \mathrm{C}$ and then was allowed to warm to rt. The mixture was concentrated under reduced pressure and $\mathrm{Et}_{2} \mathrm{O}(75 \mathrm{~mL})$ was added. The organic layer was washed with $1 \mathrm{~N} \mathrm{HCl}(75 \mathrm{~mL}), \mathrm{NaHCO}_{3}(75 \mathrm{~mL})$, and brine $(75 \mathrm{~mL})$, and then dried, filtered, and concentrated. The resulting residue was purified by column chromatography using basic alumina (3:1 hexanes/EtOAc) to afford $\mathbf{1 9 g}$ as a white crystalline solid (458 $\mathrm{mg}, 92 \%, 90 \%$ ee) with spectral data corresponding to that listed above. A combined $1.03 \mathrm{~g}$ of $90 \%$ ee material was recrystallized two times from heptane $(10 \mathrm{~mL})$ to afford $\mathbf{1 9 g}$ as a white crystalline solid (742 $\mathrm{mg}$, $72 \%, 99.6 \%$ ee). 
Determination of the absolute stereochemistry of sulfinate ester 19g. Sulfinate ester $19 \mathrm{~g}(12.1 \mathrm{mg}, 0.0383 \mathrm{mmol}, 99.6 \%$ ee) was added to a flame dried 1-dram vial containing THF $(0.200 \mathrm{~mL}, 0.200 \mathrm{M})$, and the mixture was cooled to $-78^{\circ} \mathrm{C}$. PhLi $(0.121 \mathrm{~mL}, 0.115 \mathrm{mmol}$, $0.950 \mathrm{M}$ in cyclohexane-ether) was added dropwise, and the reaction mixture was stirred for $2 \mathrm{~h}$. After the addition of $\mathrm{AcOH}(0.121 \mathrm{~mL}, 1.00 \mathrm{M}$ in THF), the mixture was warmed to rt, diluted with EtOAc $(10 \mathrm{~mL})$, and washed with brine. The aqueous layer was washed with EtOAc $(3 \mathrm{x}$ $10 \mathrm{~mL}$ ), and the combined organic extracts were dried and concentrated. The resulting residue was purified by column chromatography (1:1 hexanes/EtOAc) to afford $(R)$-tert-butyl phenyl sulfoxide $\left(6.90 \mathrm{mg}, 99 \%, 99.6 \%\right.$ ee) with spectral data corresponding to the literature report. ${ }^{3}$ HPLC (Diacel Chiralpak AS column, 90:10 hexanes/ethanol; $1.0 \mathrm{~mL} / \mathrm{min}$ ) $\mathrm{t}(S)=7.8 \mathrm{~min}$ [minor], $\mathrm{t}(R)=9.3 \min$ [major]. ${ }^{1} \mathrm{H}$ NMR $(400 \mathrm{MHz}) \delta 1.17(\mathrm{~s}, 9 \mathrm{H}), 7.47-7.52(\mathrm{~m}, 3 \mathrm{H}), 7.58-$ $7.61(\mathrm{~m}, 2 \mathrm{H})$.

\footnotetext{
${ }^{1}$ Evans, J. W.; Fierman, M. B.; Miller, S. J.; Ellman, J. A. J. Am. Chem. Soc. 2004, 126, 8134.

${ }^{2}$ Soloshonok, V. A.; Tang, X.; Hruby, V. J. Tetrahedron 2001, 57, 6375.

${ }^{3}$ Cogan, D. A.; Liu, G.; Kim, K.; Backes, B. J.; Ellman, J. A. J. Am. Chem. Soc. 1998, 120, 8011.
} 